Editorial: Hospital Metropolitano

ISSN (impreso) 1390-2989 - ISSN (electrónico) 2737-6303

Edición: Vol. 28 No 4 (2020) octubre - diciembre

DOI: https://doi.org/10.47464/MetroCiencia/vol28/4/2020/29-35

URL: https://revistametrociencia.com.ec/index.php/revista/article/view/93

Pág: 29-35

\title{
Metaplasia ósea del endometrio. Reporte de un caso y revisión de la bibliografía
}

\section{Endometrial osseous metaplasia. Case report and literatura review}

\author{
Fedra Daniela Vela Merino' iD, Hugo Marcelo Garzón Ávila² (iD, \\ Pablo Andrés Santillán Roldán ${ }^{3}$ (iD) José Fabricio Macias Zambrano ${ }^{4}$ (i)
}

\author{
Médico especialista en Ginecología y Obstetricia, ISI Mujer, Quito-Ecuador ${ }^{7}$ \\ Médico especialista en Ginecología y Obstetricia, Hospital Metropolitano, Quito- Ecuador 2 \\ Médico especialista en Anestesiología, Clínicas Atlas, Quito- Ecuador ${ }^{3}$ \\ Médico especialista en Ginecología y Obstetricia, Hospital Metropolitano, Quito- Ecuador ${ }^{4}$
}

Recibido: 20/09/2020 Aceptado: 08/10/2020 Públicado: 29/10/2020

\section{RESUMEN}

La metaplasia ósea endometrial es una patología poco común que se asocia a infertilidad secundaria. La mayoría de casos se presenta en mujeres con antecedente de gestación no evolutiva. La sospecha se hace mediante el hallazgo de un endometrio hiperecogénico que se asemeja a la imagen de un dispositivo intrauterino. El gold standard para el diagnóstico y tratamiento es la histeroscopia con el estudio histopatológico del material obtenido en el precedimeinto. La importancia de su descripción es su presentación en una paciente nuligesta. Se describe el caso clínico y revisión de la literatura.

Palabras claves: Metaplasia ósea endometrial, infertilidad secundaria, endometrio hiperecogénico.

\section{ABSTRACT}

Endometrial osseoum metaplasia is a rare condition associated with secondary infertility. Most cases occur in women with a history of non-evolutionary pregnancy. The suspicion is made by the finding of a hyperechoic endometrium that is similar of an intrauterine device. The diagnostic and therapeutic gold standard is made by hysteroscopy with histopathological study of the material obtained in the precedure. The importance of this case is its presentation in a nulliparous patient. The clinical case and literature review are described.

Keywords: Endometrial osseous metaplasia, secondary infertility, hyperechoic endometrium.

Fedra Daniela Vela Merino: Hugo Marcelo Garzón Ávila: Pablo Andrés Santillán Roldán:

José Fabricio Macías Zambrano:
IDs Orcid

https://orcid.org/0000-0001-8215-8706 https://orcid.org/0000-0003-1280-4551 https://orcid.org/0000-0001-6240-7461 https://orcid.org/0000-0002-2496-220X 


\section{Caso clínico}

Se presenta un caso clínico de metaplasia ósea endometrial en una paciente nuligesta sin antecedentes de importancia, se diagnosticó y trató de manera exitosa con histeroscopia.

\section{INTRODUCCIÓN}

La Metaplasia ósea endometrial es una rara condición. La principal afectación que causa es infertilidad secundaria. Debido a su baja incidencia no todos los médicos conocen esta patología, por lo que es importante su revisón.

\section{REPORTE DE CASO}

Se trata de una paciente femenina de 26 años, sin antecedentes clínicos o quirúrgicos de importancia, nuligesta, con ciclos menstruales regulares, fecha de última menstruación 10 días previos al ingreso. Presentó de manera espontánea expulsión de tejido aparentemente duro, lineal, de color blanco por vagina, siendo esta la causa por la que acude a consulta, el examen ecográfico revela la presencia de endometrio hiperecogénico a lo largo de toda la cavidad uterina que genera sombra posterior (Imagen 1).

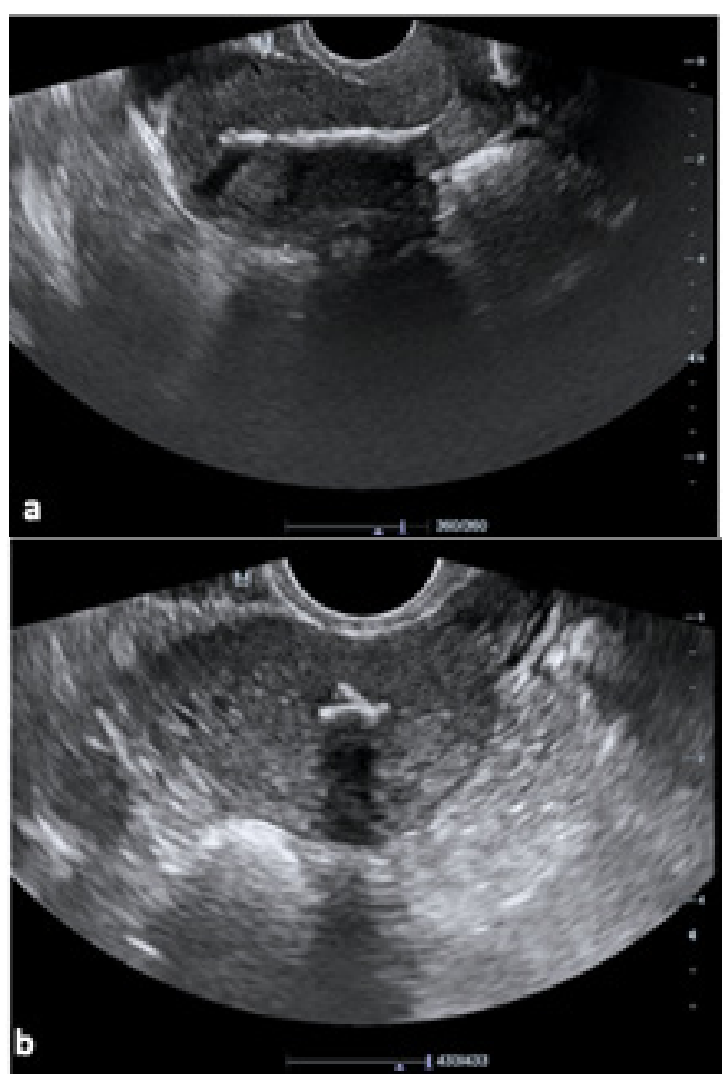

Imagen 1. Ecografía en la que se evidencia útero con endometrio hiperecogénico en toda la cavidad que genera sombra posterior, a) Corte longitudinal y b) Corte transversal.

Fuente: Imágenes obtenidas en consulta privada.
La histerosonografía con buena distención, demuestra múltiples fragmentos lineales intracavirarios hiperecogénicos que generan sombra posterior (Imagen 2).

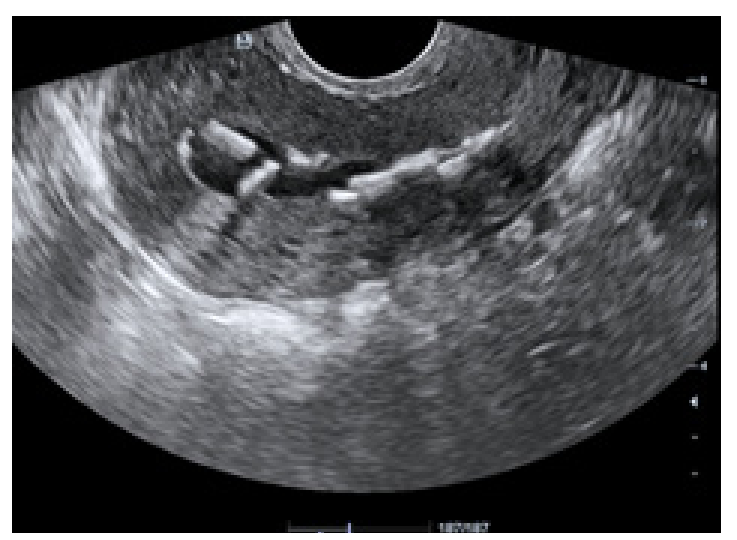

Imagen 2. Histerosonografía evidencia múltiples fragmentos lineales hiperecogénicos intracavitarios que generan sombra posterior.

Fuente: Imágenes obtenidas en consulta privada.

La impresión diagnostica del cuadro clínico se direcciona a un caso de metaplasia ósea del endometrio por lo que se programa y realiza una histeroscopia en la que se evidencian múltiples fragmentos blancos duros, algunos incrustados en la pared uterina, los cuales se extraen mediante una pinza de Grasper introducida a través del canal operatorio del histeroscopio de Bettochi (Imagen 3).
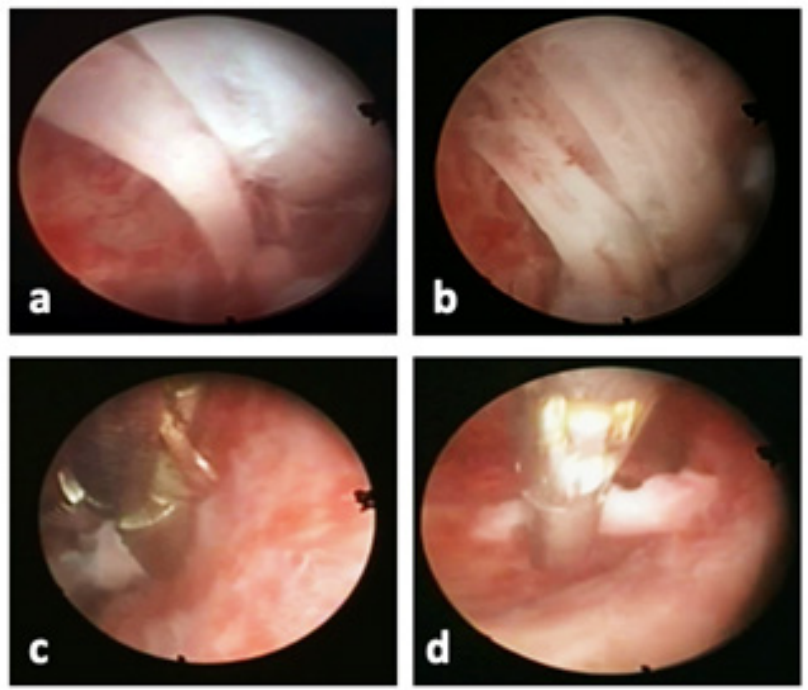

Imagen 3. Histeroscopia operatoria en la que se evidencian múltiples fragmentos blancos y duros en la cavidad endometrial. A y B) Fragmentos espiculados blancos vistos con histeroscopio. C y D) Extracción de fragmentos mediante pinza de Grasper.

Fuente: Imágenes obtenidas del video de la histeroscopia. 
El material obtenido de la histeroscopia operatoria es de aspecto óseo espiculado en múltiples fragmentos.

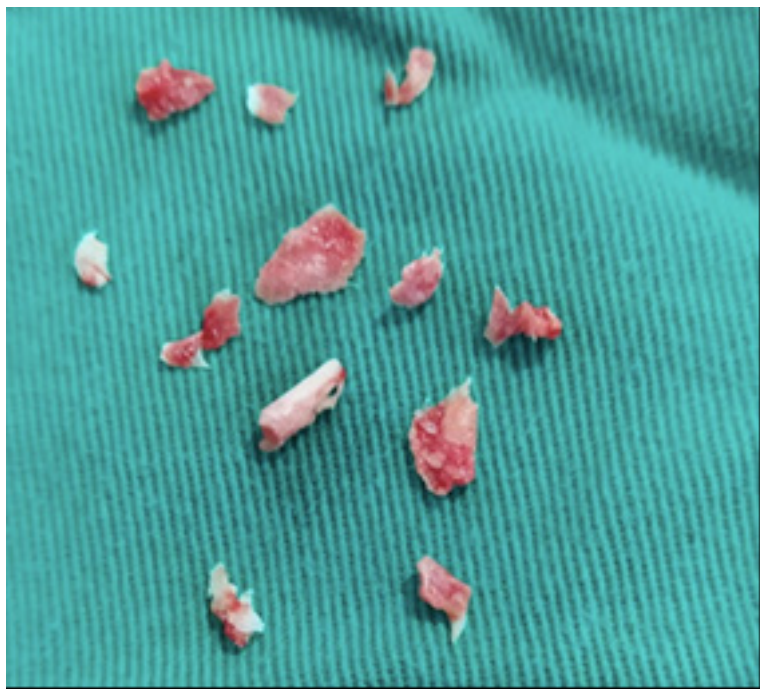

Imagen 4. Fragmentos de tejido óseo extraídos en histeroscopia.

Fuente: Foto del material obtenido en el procedimiento quirúrgico.

El material obtenido de la histeroscopia operatoria es de aspecto óseo espiculado en múltiples fragmentos.
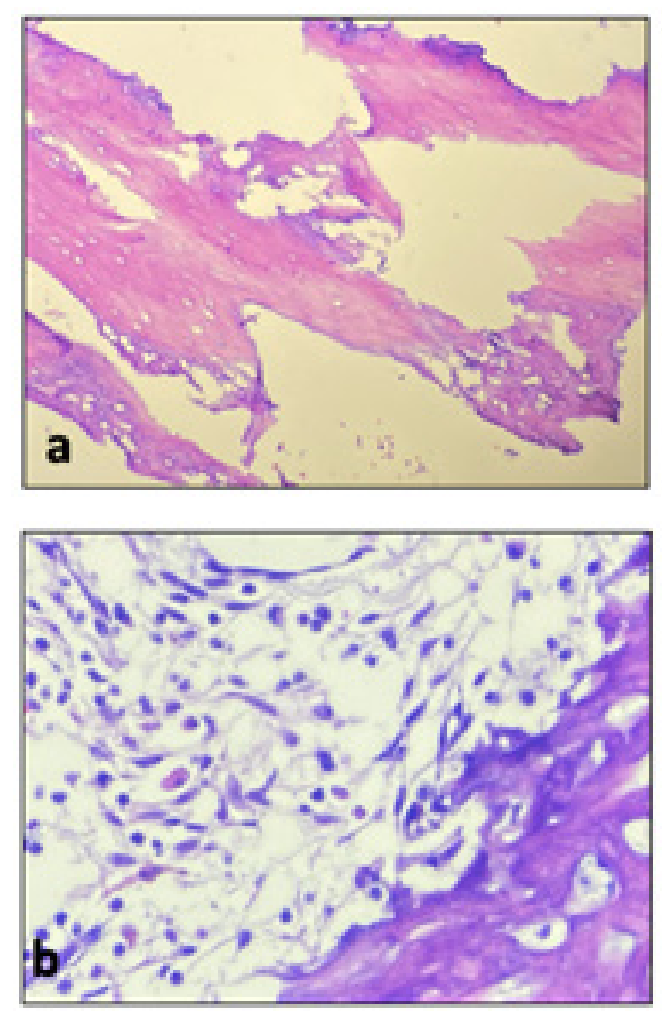

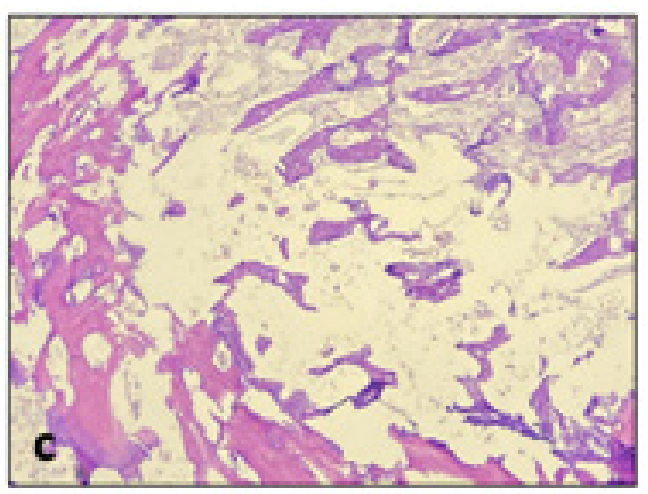

Imagen 5. Cortes histológicos que muestran: A y B) Trabéculas óseas de espesor variable bien formadas maduras englobando tejido adiposo. C) Médula ósea con incipiente hematopoyesis.

Fuente: Imágenes del laboratorio particular de Anatomía Patológica.

Se da de alta a la paciente el día del procedimiento, asintomática, con analgesia y anticoncepción. Posteriormente ha permanecido en buenas condiciones, no tiene deseos genésicos por lo que no se ha probado su fertilidad.

\section{Discusıón}

La metaplasia ósea del endometrio también se conoce como osificación endometrial, hueso intrauterino ectópico o hueso intrauterino heterotópico. Se define como una condición patológica no neoplásica endógena, ya que no se encuentra reacción tisular en el tejido endometrial estudiado, además este presenta cambios cíclicos regulares normales ${ }^{1}$. Se considera una formación de tejido óseo maduro o inmaduro a partir de un tejido distinto en un mismo individuo. En el tracto genital femenino se han descrito casos en vagina, cuello uterino, ovarios y, de manera principal en endometrio ${ }^{2,3}$.

La metaplasia ósea endometrial es una rara condición que se describió por primera vez en 1901 por el médico patólogo Mayer, en 1923 Thaler vinculó la presencia de tejido óseo en el útero a un aborto previo. En 1956 De Brux y su equipo describen por primera vez la osteogénesis en el tracto genital ${ }^{4,5}$. En 1884 Virchow atribuyó la formación ósea endometrial a la diferenciación espontánea de fibroblastos a osteoblastos ${ }^{6}$.

La incidencia estimada de esta patología es de 3 a 4 por cada 10.000 mujeres, la mayoría corresponde a un rango etario de 20 a 40 años, de las cuales un $80 \%$ tiene antecedente de gestación. Hay pocos casos descritos en mujeres nuligestas o postmenopaúsicas. Hasta la actualidad se han reportado aproximadamente 240 casos en la literatura mundial $\left.\right|^{1,7,8}$. 
El factor de riesgo más común es la presencia de una pérdida gestacional previa en segundo o tercer trimestre, ambos casos después de la formación de huesos fetales, por lo que se piensa que esta patología se debe a la implantación de partes fetales retenidas. El tiempo entre la terminación del embarazo y la identificación de la metaplasia ósea endometrial es variable, los casos descritos varían en un rango de 8 semanas a 14 años. También se puede encontrar metaplasia ósea endometrial en mujeres que consumen exceso de calcio y vitamina $D$, en alteraciones metabólicas en las que se encuentran calcificaciones metastásicas o heterotópicas, en estimulación estrogénica prolongada del endometrio o historia previa de estudios de cavidad uterina ${ }^{1}$.

La etiología exacta es incierta, se han reportado varios mecanismos patológicos como heterotopia, calcificaciones distróficas, osificación de endometritis postaborto, calcificación metastásica, metaplasia en tejido sano, terapia estrogénica prolongada después de un aborto, huesos fetales retenidos. En países como la India se recomienda descartar tuberculosis endometrial, la cual se manifiesta como presencia calcificación y osificación endometrial, lo que puede causar infertilidad ${ }^{1}$. También hay casos asociados a biopsia de endocérvix, dispositivo intrauterino, procedimientos que incluyen instrumentación en endometrio, conización cervical ${ }^{9}$.

Las investigaciones respecto al origen del tejido óseo son contradictorias. En algunos estudios se ha demostrado mediante análisis de ADN que el tejido óseo presente no tiene material fetal, por lo que se piensa que es una verdadera metaplasia de las células del estroma endometrial por elementos pluripotentes3,4. Sin embargo en el 2008 se publicó un estudio de un caso en el que el estudio genético evidenció alelos que no estaban presentes en la paciente, por lo que se concluyó que el origen del tejido óseo fue fetal ${ }^{10}$.

En cuanto a la fisiopatología se cree que cualquier entidad que cause endometritis crónica puede estimular la proliferación de células mesenquimatosas que tienen la propiedad inherente de metaplasia, y se pueden diferenciar en condroblastos u osteoblastos $^{8}$. También se ha descrito la teoría de un sistema de radical superóxido dismutasa que pueda ser funcional en la metaplasia ósea ya que juega un papel importante en la diferenciación endometrial, la inflamación crónica debida a la retención de tejidos gestacionales puede promover la liberación de radical superóxido o factor de necrosis tumoral de los fagocitos. El endometrio deficiente en la actividad protectora de la superóxido dismutasa podría presentar una agresión duradera a las células estromales multipotenciales lo que puede ocasional la transformación de estas células en osteoblastos ${ }^{8}$.
Clínicamente puede ser asintomática (con ciclos regulares) o presentarse como dolor pélvico, irregularidad menstrual, dismenorrea, dispareunia, sangrado uterino anormal o secreción vaginal, sin embargo la presentación más común es infertilidad secundaria ${ }^{3,11,12}$. En raras ocasiones ocurre la expulsión espontánea de material óseo por la vagina, lo cual se considera patognomónico ${ }^{7}$.

La infertilidad se explica por tres mecanismos 5,13,14:

- Efecto mecánico: Prevención de la implantación por obliteración de la cavidad uterina.

- Efecto de dispositivo intrauterino: Inflamación crónica debido a los fragmentos óseos, lo que impide la implantación.

- Toxicidad embrionaria: Toxicidad embrionaria directa debido a las partículas óseas $5,13,14$

La sospecha diagnóstica se hace mediante ultrasonido, en ciertos casos se puede recurrir a una tomografía. Se ha visto que la resonancia magnética y la histerosalpingografía no son adecuados para esta patología ${ }^{3}$.

Los hallazgos ecográficos más comunes son bandas hiperecogénicas únicas o múltiples en la cavidad uterina con sombra acústica (Imagen 6), puede tener apariencia similar a la de un dispositivo intrauterino ${ }^{4}$.

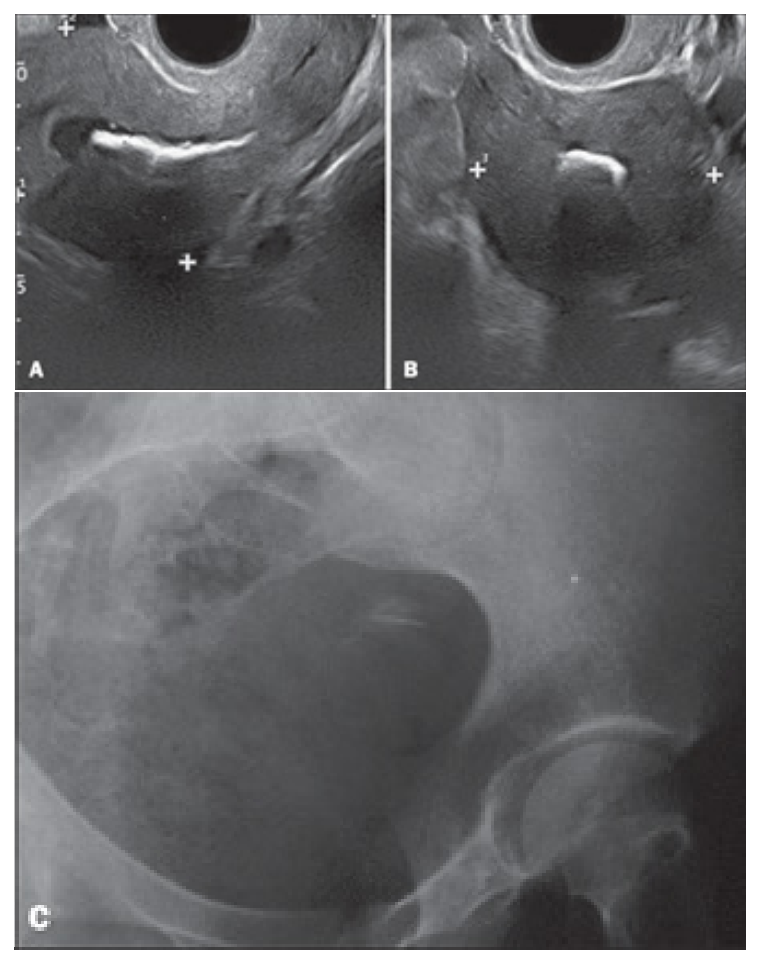

Imagen 6. Imágenes de un caso de metaplasia ósea endometrial.

A) Corte longitudinal B) Corte transversal de ecografía transvaginal que evidencia zona hiperecogé- 
nica en el endometrio con sombra acústica posterior compatible con calcificaciones. C) Radiografía de cadera en vista oblicua, se observa una imagen con densidad de calcificación correspondiente a la encontrada en la ecografía ${ }^{15}$.

Fuente: Luiz Felipe Alves Guerra, Laís Bastos Pessanha, Gabriel Antonio de Oliveira, Adriana Maria Fonseca de Melo, Flavia Silva Braga RSM de S. Endometrial osseous metaplasia: sonographic, radiological and histopathological findings. Radiol Bras 2016;49(8):62-3.

En la histerosonografía puede haber defectos de llenado por las espículas óseas ${ }^{4}$. En ocasiones no se distiende cuando a la colocación de solución salina, lo cual puede causar dolor a la paciente ${ }^{16}$.

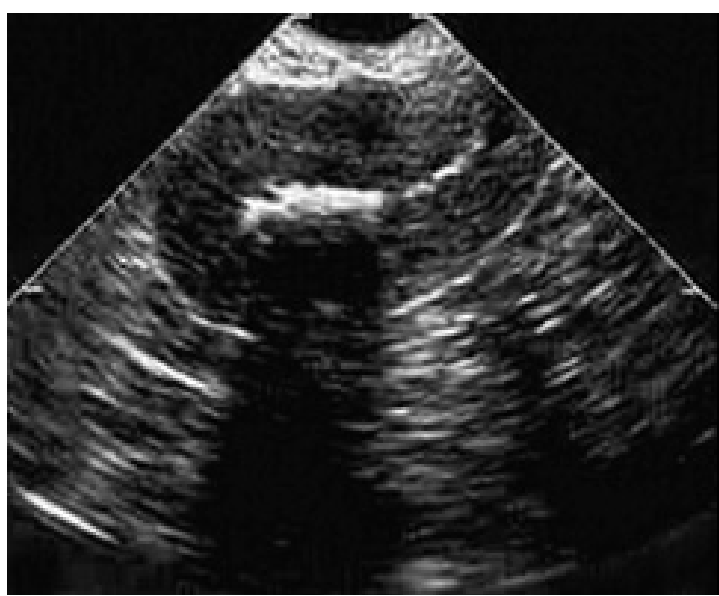

Imagen 7. Caso clínico de metaplasia ósea en la que se observó área hiperecogénica en la cavidad endometrial con una extensa sombra posterior. Al intentar infundir con solución salina no se produjo distención de la cavidad y resultó en dolor intenso ${ }^{16}$.

Fuente: Killick SR. Ultrasound assessment of fertility. In: Clinical Ultrasound [Internet]. Thrid Edit. Elsevier Ltd; 2011. p. 730-9.

Cuando se evidencian hallazgos ecográficos sugerentes, se debe recurrir a la histeroscopia ${ }^{3}$. En ésta se observa una red blanca de espículas blanquecinas, que frecuentemente surgen de la pared posterior y se extienden perpendicularmente hacia la cavidad, con una consistencia táctil dura (Imagen 8). En ocasiones se pueden encontrar huesos fetales bien formados ${ }^{4}$.
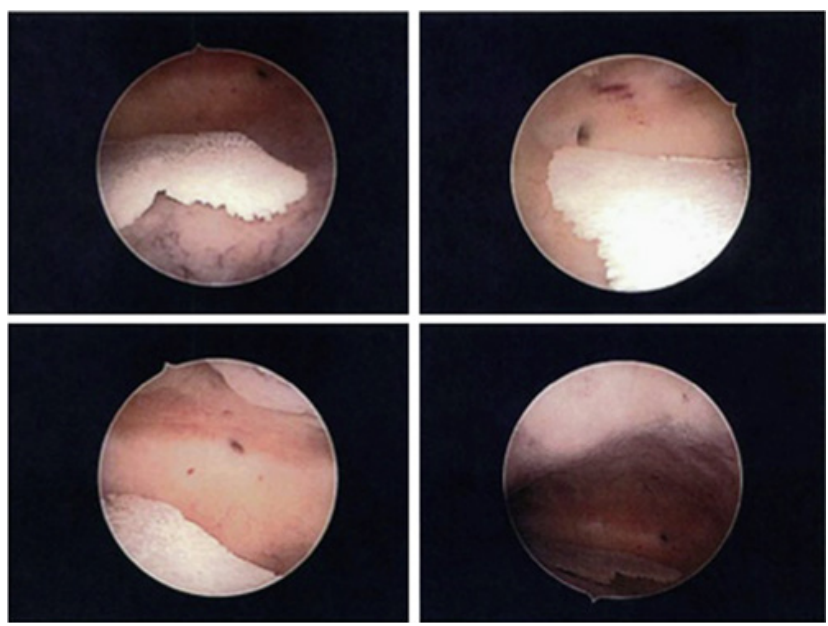

Imagen 8. En histeroscopia de oficina se evidencia material blanco poroso que flota en la cavidad uterina $^{17}$.

Fuente: Roach MK, Thomassee MS. An incidental finding of endometrial osseous metaplasia during office hysteroscopy. Am J Obstet Gynecol 2015;212(3): 402.e1-402.e2.

La confirmación diagnóstica se realiza mediante examen histopatológico en el que se evidencia tejido de características óseas (Imagen 9). Se puede diferenciar los casos de metaplasia ósea de la retención de tejido fetal por la ausencia de reacción del tejido circundante y de osificación encondrial ${ }^{4}$. La biopsia suele mostrar reacción tisular mínima o nula, lo que explica que la osificación es de naturaleza distrófi$\mathrm{ca}^{1}$.

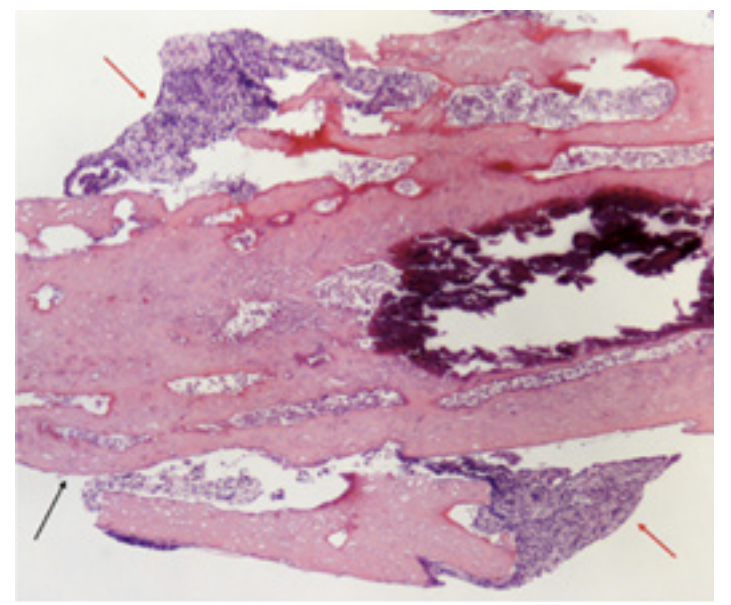

Imagen 9. Tejido óseo trabeculado maduro (flecha negra) de origen metaplásico rodeado por un endometrio de apariencia normal (flechas rojas) (tinción de hematoxilineosina 40) ${ }^{18}$.

Fuente: Giannella L, Gelli MC, Ippolito GD, Ciavattini A. Ultrasound appearance of endometrial bone metaplasia mimics an intrauterine device. Am J Obstet Gynecol 2020;223(4):580-1. 
Es importante hacer diagnóstico diferencial con ciertas entidades que pueden ser similares, entre las cuales están ${ }^{1,9,11,19}$ :

- Retención de un dispositivo intrauterino y calcificación.

- Tumores Müllerianos mixtos.

- Gliosis uterina con calcificación.

- Teratomas uterinos.

- Tuberculosis endometrial.

- Tumores mesodérmicos mixtos.

- Endometritis calcificante asociada a síndrome de Asherman.

- Retención prolongada de huesos fetales con reacción tisular y osificación endocondral ${ }^{1,9,11,19}$.

De inicio se recurría a la dilatación y legrado para el diagnóstico y tratamiento de estos casos. Actualmente la histeroscopia es el gold standard con fines diagnósticos y terapeúticos. En estos casos no se requiere de preparación endometrial con anticonceptivos o análogos de GNRH ya que la proliferación endometrial es escasa ${ }^{20}$. El tratamiento se realiza mediante escisión histeroscópica o resectoscópica, la mayoría logra resolución sin necesidad de una segunda evacuación de la cavidad. En casos de metaplasias óseas extensas con invasión profunda del miometrio se puede usar guía ecográfica - laparoscópica. Se ha estudiado poco acerca de los residuos óseos incrustados en el miometrio, sin embargo al parecer no tienen importancia siempre y cuando no se extiendan hacia la cavidad ${ }^{4}$. En el 2019 se realizó una publicación en la que describen un caso de metaplasia ósea resuelto mediante técnica de litotricia uterina transcervical, lo cual en teoría disminuye el riesgo de perforación uterina ${ }^{11}$.

Posterior al procedimiento para extraer el tejido óseo, se recomienda usar estrógeno conjugado por 6 a 8 semanas para favorecer la proliferación endometrial ${ }^{11}$.

En cuanto al pronóstico reproductivo, un antecedente de osificación endometrial parece no afectar el resultado del embarazo ${ }^{4}$. Un estudio del 2009 con cinco pacientes que presentaron infertilidad o abortos recurrentes asociados a metaplasia ósea endometrial a las que se les realizó una histeroscopia con remoción de los fragmentos se evidenciaron tres mujeres presentaron embarazo espontáneo en un periodo de tiempo de 4 a 16 meses, mientras que una paciente recurrió a reproducción asistida sin resultado favorable. Una paciente abandonó el estudio ${ }^{21}$.

\section{CONCLUSIONES}

- La metaplasia ósea endometrial es una patología rara y poco conocida que causa infertilidad secundaria.

- La sospecha se hace con la historia clínica y exámenes complementarios como la ecografía y histerosonografía.

- Una vez que se sospeche en un caso de metaplasia ósea endometrial se debe realizar una histeroscopia con fines diagnósticos y terapeúticos.

- Es necesario enviar el material obtenido en la histeroscopia a estudio histopatológico para determinar el tipo de tejido encontrado.

- Luego del procedimiento quirúrgico se debe estimular la proliferación endometrial mediante terapia hormonal.

- Se requieren estudios de buena calidad para determinar el pronóstico reproductivo de las pacientes con metaplasia ósea endometrial.

\section{CONTRIBUCIÓN DE LOS AUTORES}

a. Concepción y diseño del trabajo: Daniela Vela Merino, Hugo Garzón Ávila

b. Recolección / obtención de resultados: Daniela Vela Merino, Hugo Garzón Ávila, Pablo Santillán Roldán

c. Análisis e interpretación de datos: Fabricio Macías Zambrano, Pablo Santillán Roldán

d. Redacción del manuscrito: Daniela Vela Merino, Hugo Garzón Ávila, Fabricio Macías Zambrano, Pablo Santillán Roldán

e. Revisión crítica del manuscrito: Hugo Garzón Ávila

f. Aprobación de su versión final: Hugo Garzón Ávila

g. Aporte de pacientes o material del estudio: Hugo Garzón Ávila

h. Obtención de financiamiento: No corresponde

i. Asesoría estadística: No corresponde

j. Asesoría técnica o administrativa: Hugo Garzón Ávila

k. Otras contribuciones: No corresponde

\section{CONFLICTO DE INTERESES}

Los autores declaran no tener ningún conflicto de intereses personal, financiero, intelectual, económico y de interés corporativo con el Hospital Metropolitano y los miembros de la Revista Metrociencia. 


\section{FINANCIACIÓN}

No corresponde.

\section{AGRADECIMIENTOS}

Los autores del presente trabajo agradecen a los profesionales involucrados en el caso que han proporcionado información e imágenes.

\section{REFERENCIAS BIBLIOGRÁFICAS}

1. T Umashankar, Shobhana Patted RH. Endometrial osseous metaplasia: Clinicopathological study of a case and literature review. J Hum Reprod Sci. 2010;3(2):102-5

2. Patil SB, Narchal S, Paricharak DG MS. Endometrial Osseous Metaplasia : Case Report with Literature Review. Ann Med Health Sci Res. 2013;3(November):10-2.

3. Miriam S. Krause, Henry Bohler STN. Saline Infusion Sonogram is Superior to CT Scan for Diagnosis of Osseous Metaplasia. Fertil Steri [Internet]. 2012;97(3):S21. Available from: http://dx.doi.org/10.1016/j. fertnstert.2012.01.05

4. Onderoglu LS, Yarali H, Gultekin M, Katlan D, D M. Endometrial osseous metaplasia: an evolving cause of secondary infertility. Ferti Steril [Internet]. 2008;90(5):2013-5. Available from: http://dx.doi.org/10.1016/j.fertnstert.2008.01.001

5. Lainas T, Ph D, Zorzovilis I, Petsas G, Ph D. Osseous metaplasia case report and review. Fertil Steril. 2004;82(5):1433-5.

6. J C Rosa e Silva JC, Barcelos ID, Navarro PA, Ferriani RA. Osseous metaplasia of the endometrium associated with infertility: A case report and review of the literature. J Med Case Rep. 2009;3(September):3-7.

7. Alba Lucía Mondragón-Cedeño, Ángel David Miranda-Cruz, Sergio Augusto Parra-Duarte, Eliana Paredes-Becerra AMZ-C. Metaplasia ósea endometrial: reporte de casos y revisión de la literatura. Rev Colomb Obstet Ginecol. 2011;62(3):255-60.

8. Garima Gupta, Divya Mahindru, Aparajita D'Souza SG. Osseous Metaplasia of Endometrium : A Very Rare Entity. World J Laparosc Surg. 2017;10(August):69-72.

9. Valter Feyles, Terence Moyana RP. Recurrent pregnancy loss associated with endometrial hyperechoic areas (endometrial calcifications ): A case report and review of the literature. Clin Exp Obstet Gynecol. 2000
10. Tonin PN, Ph D, Arcand SL, Sc M. Calcified tissue of fetal origin in utero. Fertil Steril. 2008;89(1):217-8.

11. Akanksha Sood, Emilie Lewis, Ben Grey LM. Novel Management of Endometrial Osseous Metaplasia. World J Surg Surg Res. 2019;2:1-2.

12. Madhu Chaturvdi AS. Intrauterine bony fragments - An unexpected finding in the hysterectomy specimen. Autops Case Reports. 2020;(Figure 3):3-6.

13. Bosch $T$ Van den. Favorable pregnancy outcome in a woman with osseous metaplasia of the uterus. Ultrasound Obstet Gynecol. 2000; 15:445-6

14. Marcus SF, Bhattacharya J, Rdms GW, Brinsden P, Hamou J. Endometrial ossification : A cause of secondary infertility Report of two cases. Am J Obstet Gynecol [Internet]. 1994;170(5):1381-3. Available from: http://dx.doi.org/10.1016/S0002-9378(13)90472-5

15. Luiz Felipe Alves Guerra, Laís Bastos Pessanha, Gabriel Antonio de Oliveira, Adriana Maria Fonseca de Melo, Flavia Silva Braga RSM de S. Endometrial osseous metaplasia: sonographic, radiological and histopathological findings. Radiol Bras. 2016;49(8):62-3.

16. Killick SR. Ultrasound assessment of fertility. In: Clinical Ultrasound [Internet]. Thrid Edit. Elsevier Ltd; 2011. p. 730-9. Available from: http://dx.doi.org/10.1016/B978-0-7020-3131-1.00038-9

17. Roach MK, Thomassee MS. An incidental finding of endometrial osseous metaplasia during office hysteroscopy. Am J Obstet Gynecol [Internet]. 2015;212(3):402.e1-402.e2. Available from: http://dx.doi. org/10.1016/j.ajog.2014.11.015

18. Giannella L, Gelli MC, Ippolito GD, Ciavattini A. Ultrasound appearance of endometrial bone metaplasia mimics an intrauterine device. Am J Obstet Gynecol [Internet]. 2020;223(4):580-1. Available from: https://doi.org/10.1016/j.ajog.2020.01.023

19. Togas Tulandi, Mohammed Al-Sunaidi, Jocelyne Arseneau, Patricia Tonin SA. Calcified tissue of fetal origin in utero. Fertil Steril. 2008;89(1):2007-8.

20. Alejandro Manzur SV. Extensa metaplasia ósea endometrial y exitoso embarazo espontáneo post tratamiento. Casos clínicos. Rev Med Chile. 2010;138:1004-7.

21. Deffieux X, Lousquy R, Faivre E FH. Fertility after Hysteroscopic Management of Endometrial Osseous Metaplasia. J Minim Invasive Gynecol. 2009;16:60. 